\title{
Lues, bist du es?
}

\author{
Ein zuvor völlig gesunder 25-jähriger \\ Mann stellte sich wegen multipler, \\ nicht juckender scharf begrenzter \\ erythematöser und leicht schup- \\ pender Plaques an den Handflächen \\ und Fußsohlen vor.
}

- Abgesehen von den Hautveränderungen fühlte er sich wohl, die körperliche Untersuchung war unauffällig. Auch auf Befragen verneinte er, eine ulzerierende Läsion am Genitale gehabt zu haben. Allerdings gab er mehrere ungeschützte Sexualkontakte mit verschiedenen Partnerinnen an.

Ein VDRL-Test war mit einem Titer von 1:64 positiv, ein Mikrohämagglutinationsassay auf Antikörper gegen Treponema pallidum reaktiv, sodass die Diagnose einer sekundären Syphilis gestellt werden konnte. Serologische Untersuchungen auf Chlamydien, Gonorrhö und HIV verliefen negativ. Der Patient wurde mit einer Einmalgabe von 2,4 Millionen IU Penicillin G behandelt, worauf sich das Exanthem rasch zurückbildete.
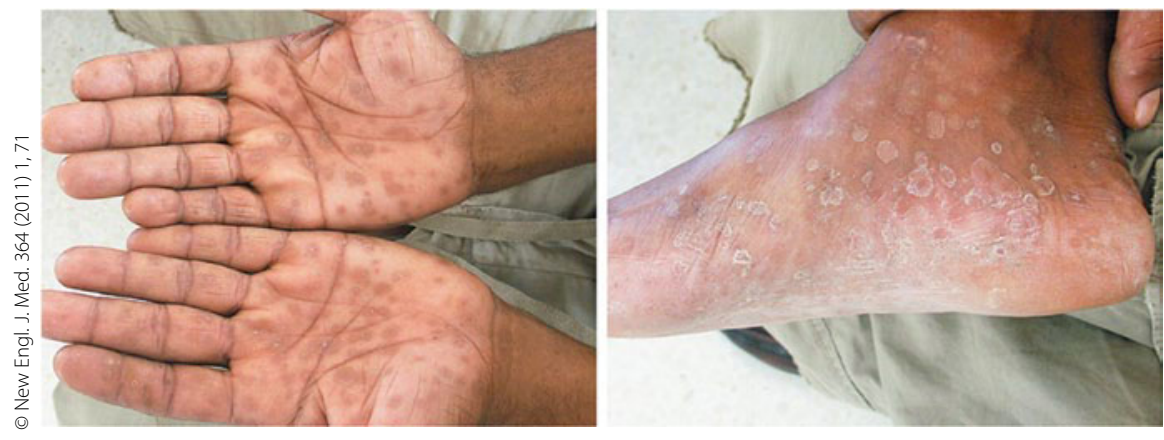

Erythematöse Plaques an den Handflächen und Fußsohlen.

\section{Kommentar}

Die sekundäre Syphilis (Lues II) ist durch die hämatogene und lymphogene Aussaat des Erregers bedingt und beginnt 4-10 Wochen nach der Infektion. Sie kann eine "bunte" klinische Symptomatik aufweisen und nahezu alle dermatologischen Krankheiten imitieren. Daher hat man sie früher auch als den "Affen der Dermatologie" bezeichnet und daher rührt auch der alte Kalauer erfahrener Kliniker: "Lues, bist du es?" Neben allgemeinen Krankheitszeichen wie Fieber, Müdigkeit, Kopf-, Gelenk- oder Muskelschmerzen kommt es zu spezifischen Exanthemen und
Enanthemen, sog. Syphiliden, die allerdings eine hohe Variabilität aufweisen. Typischerweise tritt erst ein stammbetontes, oft kaum erkenntliches masernähnliches Exanthem ohne Juckreiz auf, das eine Pityriasis rosea, eine Psoriasis, ein Arzneimittelexanthem aber auch ein akutes Virusexanthem imitieren kann. Besonders typisch sind Syphilide der Hohlhand oder der Fußsohlen (Palmoplantarsyphilide).

H. S. FÜESSL "

\section{- F. Badri, S. B. Jennet}

(Korr.: Habib Thameur Hospital of the University of Tunis El ManarTunis; talel_badri@yahoo. fr) Rash associated with secondary syphilis. New Engl. J. Med. 364 (2011) 1, 71

\section{Keime mögen elektronische Wasserhähne}

\section{Elektronische Wasserhähne sollen einerseits den Wasserverbrauch senken und andererseits die Gefahr einer Kontamination der Hände mit Keimen reduzieren.}

- Gerade in Krankenhäusern ist es wichtig, eventuelle Kontaminationsquellen auszuschalten. Für immunsupprimierte Patienten können die Keime lebensgefährlich werden. Wissenschaftler der Johns Hopkins University in Baltimore (USA) gingen jetzt der Frage nach, ob Wasser aus elektronischen Wasserhähnen wirklich weniger Keime enthält als das aus mechanischen, die manuell bedient werden müssen.

Wasserproben von 20 neu installierten, elektronischen Hähnen und 20 manuellen Hähnen in drei Hospitalabteilungen wurden gesammelt. Die manuellen und die elektronischen Hähne waren jeweils in benachbarten Räumen angebracht und hatten jeweils die gleiche Wasserquelle. Die Wasserproben wurden auf bakterielle Kontamination untersucht. Ebenfalls untersucht wurde der Effekt einer Chlorindioxiddesinfektion der Wasserhähne.

Das wichtigste Ergebnis war, dass in $54(50 \%)$ der 108 Wasserkulturen von elektronischen Hähnen Legionellen wuchsen, verglichen mit 11 (15\%) von 75 Wasserkulturen von manuellen Hähnen $(\mathrm{p}<0,001)$. Chlorindioxid desinfizierte elektronische Hähne schlechter als die manuellen und niemals vollständig.

\section{Kommentar}

Elektronische Hähne sind stärker mit Legionellen und anderen Bakterien kontaminiert als manuelle. Das liegt möglicherweise am Aufbau der sensorgesteuerten Hähne, der das Bakterienwachstum durch eine größere Innenfläche fördert. Eine Desinfektion war nicht möglich. Die elektronischen Hähne wurden deshalb aus der Klinik wieder entfernt.

K. MALBERG =

- E. R. M. Sydnor et al.

Electronic-eye faucets: Help or hindrance to infection control and prevention? Society for Health Care, Epidemiology of America, Annual scientific meeting 2011, April 1-4, Dallas, Texas 